\title{
The Erroneous Appearance of Silver Nitrate on CT Imaging in Patients with Severe Otitis Externa; A Case Series
}

\author{
Hafsa Javed ${ }^{1}$, Salman Hashmi ${ }^{1}$, and Asad Qayyum ${ }^{1}$ \\ ${ }^{1}$ North West Anglia NHS Foundation Trust
}

January 9, 2022

\begin{abstract}
Introduction Silver nitrate is commonly used within otolaryngology to treat granulation tissue in severe otitis externa. It appears radio-opaque on CT (computed tomography) imaging and therefore can mimic bony fragments and foreign bodies. This is particularly cumbersome when the phenomena correlates to the clinical complaint. Discussion We report two cases of 73-year-old and 75-year-old males who presented with chronic otalgia and discharge. Granulation tissue in the external auditory canal was identified and chemically cauterised with silver nitrate. Subsequent CT petrous bones demonstrated an unidentified foreign body in the canal with extensive soft tissue swelling giving an impression of a wick in situ and "minor bony erosion in the left external acoustic canal" respectively. An additional CT of a 57-year-old female who had been treated with silver nitrate for granulomatous tissue reported "multiple highly radiopaque foci in the external auditory canal, suggestive of foreign body". Though relatively unknown, this phenomenon has been reported in literature. However, there are few reports of silver nitrate artefacts in $\mathrm{CT}$ images of the head no cases in the context of otitis externa. Our patients avoided further imaging or surgery following clarification with the radiologists and symptomatic improvement with long-term intravenous antibiotics. Conclusion Given the prevalence of CT imaging and cauterization in otolaryngology, we recommend contemporaneously documenting the use of silver nitrate and highlighting this on request forms to avoid alarming erroneous reports, unnecessary investigation and surgical procedures. We also recommend, where clinically acceptable, to use silver nitrate prior to imaging.
\end{abstract}

\section{The Erroneous Appearance of Silver Nitrate on CT Imaging in Patients with Severe Otitis} Externa; A Case Series

\section{Key Points}

1. Silver nitrate has a high mass attenuation coefficient

2. Silver nitrate appears radio-opaque on CT imaging of the head

3. Silver nitrate can mimic foreign bodies or bony fragments on CT head imaging

4. Patients should be imaged prior to silver nitrate use if indicated

5. The use of silver nitrate must be clearly documented contemporaneously in CT head request forms

\section{Introduction}

Silver nitrate has been used in surgical practice for centuries. It was first described in 1829 by Hugginbottom for the management of inflammatory wounds and ulcers. ${ }^{1}$ Silver nitrate is an inorganic compound which, when exposed to water, ionizes into free silver ions which act on tissues and blood vessels to cause coagulation, necrosis and thrombus fromation. ${ }^{2}$ Its antiseptic action is primarily related to apoptosis leading to cell death. ${ }^{2}$ Because of its antiseptic and wound healing properties, it is commonly used as a topical agent for cauterizing bleeding points particularly over mucosal surfaces, burning granulations and even cauterizing non-healing ulcers for acceleration of the healing process. ${ }^{3}$

Granulations are considered to be one of the diagnostic criteria in necrotizing otitis externa (NOE). ${ }^{4}$ These 
lesions are common and are often managed by direct application of silver nitrate especially after taking biopsies due to use its dual effect. ${ }^{1}$ Silver nitrate not only provides haemostasis but also inhibits fibroblast proliferation resulting in rapid resolution of granulation tissues. ${ }^{3}$

The compound appears densely radio-opaque on computerized tomography (CT) imaging mimicking bony fragments and foreign bodies. This is particularly cumbersome when bony erosion is most relevant in diagnosis. Despite its regular use in NOE, it is not widely known to be present as a confusing artifact in cross-sectional imaging. Medical literature highlights a few cases in which this phenomenon is described; however, this is predominantly within plain radiographs. There are also no reports in the context of NOE.

\section{Methods}

We report three cases within our large District General Hospital (DGH) in which the appearance of silver nitrate was initially incorrectly identified in radiological reporting as either a foreign body or bony fragments in patients with otitis externa (OE) or NOE. The cases have been reported using CARE guidance following written consent from all patients. ${ }^{5}$

\section{Case Series}

A 74-year-old male with severe left sided OE presented to the emergency department (ED) with worsening left sided otalgia and external auditory canal (EAC) granulations. This was on the background of insulin dependent type-2 diabetes mellitus (T2DM) and hypertension (HTN). A CT of the petrous bones was requested. In the interim, granulation tissue in the external ear was cauterized with silver nitrate. The Radiologist described a marked bony erosion and the subsequent report demonstrated "perforation of the tympanic membrane, opacification of the tympanic cavity with partial erosion of the ossicles" (Figure 1). Left sided malignant $\mathrm{OE}$ and acute mastoiditis were suspected and therefore the patient was admitted and commenced intravenous tazobactam and piperacillin (Tazocin). On further clinical correlation and discussion between the head and neck radiologist and consultant otolaryngologist, bony erosion, perforation and intracranial extension were ruled out. A diagnosis of left sided otitis externa was made, and the patient completed an extended course of intravenous outpatient antibiotic therapy (OPAT) to prevent progression to malignant otitis externa.

Similarly, another patient, a 75-year-old male, was admitted with suspected left sided malignant OE on the background of long-standing OE. He also had a history of T2DM on insulin and HTN. Over-granulation tissue noted in his ear canal was cauterized with silver nitrate. A subsequent CT reported an unidentified foreign body in the canal with extensive soft tissue swelling giving an impression of a wick in situ. (Figure 2). Again, on further clarification between the consultants in both departments, it was concluded the CT did not show eroded bone, rather there appeared to be an artefact in the images as a result of silver nitrate usage. The patient had already been treated with intravenous Tazocin and so was discharged with a long course of ciprofloxacin and meropenem.

The final patient was a 58-year-old female with recurrent OE and a history of previous eustachian tube dysfunction with tuboplasty in 2013. She presented to clinic with an 18-month history of right sided otalgia and discharge. Swabs were positive for achromobacter xylosoxidans. Despite multiple courses of topical antifungals (betamethasone and clotrimazole) and oral antibiotics during these 18 months, the symptoms had not resolved and therefore she underwent a CT head for suspected necrotizing OE. Alarmingly, the CT reportedly identified "multiple highly radiopaque foci in the external auditory canal, suggestive of a foreign body" (Figure 3). Clinical examination prior to and following CT imaging did not reveal any foreign bodies however, there was evidence of recent silver nitrate use to treat over-granulation tissue as well as eczematic skin within the ear canal. The patient was treated for acute OE with local and systemic antibiotics and made a complete recovery.

\section{Discussion}

We have reported three cases in which $\mathrm{CT}$ imaging of patients with OE/NOE was wrongly interpreted due to the use of silver nitrate. This is important as skull base bone erosion is the first confirmatory radiologic sign 
of its progression towards NOE. ${ }^{4}$ Silver nitrate is commonly used in these patients and often imaging follows. This can lead to misinterpretation, potentially wrong diagnoses and hence more aggressive treatment.

CT is a quick and readily available imaging modality which can be used for diagnostic purposes in otology. Among its many uses in medicine; it is vital in differentiating otitis externa with its necrotizing counterpart. As both patients with OE and NOE frequently present with granulations which are cauterized with silver nitrate there is a risk the appearance of silver nitrate can be misinterpreted.

The radiopacity of silver nitrate can be attributed to its silver cation. As a metal its mass attenuation coefficient is described as high as 14 times that of cortical bone. ${ }^{3}$ This explains why only a thin layer over granulation tissues is mistaken for bone within the EAC as it is a narrow canal surrounded by bone. Further doubt is created in the context of NOE due to surrounding soft tissue swelling.

Our literature review revealed ten other cases in which silver nitrate artefacts were seen on imaging. However, only one of otologic origin was recently reported by Godse et al. ${ }^{6}$ They reported a case of a patient with EAC squamous cell carcinoma who presented with granulations which were biopsied and cauterized for haemostasis. A subsequent $\mathrm{CT}$ scan revealed a foreign body in EAC which was later attributed to be silver nitrate application.

Among other otolaryngology cases, Livingstone et al. reported silver nitrate artefact being misconstrued as a foreign body on CT imaging within pharyngeal mucosa after it was used for coagulation following incision and drainage for a peritonsillar abscess. ${ }^{7}$ Likewise, Johnston et al. reported a case in which a patient underwent a revision bilateral Functional Endoscopic Sinus Surgery (FESS) after foreign bodies were noted in her paranasal sinuses by a CT of the sinuses. ${ }^{15}$ The foreign body was later identified as silver nitrate that had been used for recurrent epistaxis on a number of occasions in the ED. ${ }^{8}$

Further literature review highlighted a case in which wrongfully, a bony fragment in a supracondylar humeral fracture was reported on plain radiographs in the absence of clinical correlation. ${ }^{3}$ Healy et al. reported two cases in which silver nitrate was incorrectly identified as a foreign body on plain radiographs of the fingers. ${ }^{9}$ A similar case was described by Peter $\mathrm{Vu}$ et al., in which the patient unfortunately underwent surgical intervention due to the artefactual appearance of fingertip heterotopic ossification. ${ }^{10}$

\section{Conclusion}

Given the prevalence of CT imaging and cauterization in otologic practice, especially in context of OE and NOE, we recommend imaging patients before silver nitrate cautery if required. If imaging is delayed we recommend contemporaneously documenting the use of silver nitrate and highlighting this on request forms to avoid alarming erroneous reports, unnecessary investigation and surgical procedures. We also encourage discussions between otolaryngology and radiology departments to educate all physicians on the potential appearance of silver nitrate on CT imaging.

\section{References}

1. Higginbottom J. A practical essay on the use of the nitrate of silver in the treatment of inflammation, wounds, and ulcers. London (GB): John Churchill \& Sons; 1865.

2. Kunio NR, Schreiber MA. Topical hemostatic agents. In: consultative hemostasis and thrombosis. 2nd ed. Oregon (US): Elsevier Inc. 2013. p538-545.

3. Narayan N, Nogaro M, Littlewood A, Latimer M. The misleading appearance of silver nitrate on plain radiography. Ann R Coll Surg Engl. 2014;96(3):e3.

4. Cohen D, Friedman P. The diagnostic criteria of malignant external otitis. J Laryngol Otol. 1987;101(3):216-221.

5. Gagnier JJ, Kienle G, Altman DG, Moher D, Sox H, Riley D; the CARE Group. The CARE Guidelines: Consensus-based Clinical Case Reporting Guideline

6. Godse NR, Branstetter BF, Hobson CE. Silver nitrate: A potential mistaken foreign body. Otol Neurotol 2019;40(8):e850-851. 
7. Livingstone D, Alghonaim Y, Jowett N, Sela E, Mlynarek A, Forghani R. Silver nitrate mimicking a foreign body in the pharyngeal mucosal space. World J Radiol 2015;7(5):100.

8. Johnston JJ. Silver nitrate in the sphenoid sinus following nasal cauterization. Clin Med Rev Case Reports 2018;5(3).

9. Healy C, Canney M, Murphy A, Regan P. Silver nitrate masquerading as a radiopaque foreign body Emerg Radiol 2007;14(1):63-64.

10. Vu P, Faraday R, Vu D, Kim J. Silver nitrate stain masquerading as a heterotopic ossification. Radiol case reports 2020;15(5):450-453.

Figure 1: Left sided silver nitrate artefact appearing as a bony fragment in left EAC due to similar densities of the silver nitrate and bone.

Figure 2: Silver nitrate appearing as a metallic foreign body in left EAC.

Figure 3: Right EAC granulations and multiple overlying metallic spots.

\section{Hosted file}

Figure 1 Silver Nitrate.docx available at https://authorea.com/users/454652/articles/552186the-erroneous-appearance-of-silver-nitrate-on-ct-imaging-in-patients-with-severe-otitisexterna-a-case-series

\section{Hosted file}

Figure 2 Silver Nitrate.docx available at https://authorea.com/users/454652/articles/552186the-erroneous-appearance-of-silver-nitrate-on-ct-imaging-in-patients-with-severe-otitisexterna-a-case-series

\section{Hosted file}

Figure 3 Silver Nitrate.docx available at https://authorea.com/users/454652/articles/552186the-erroneous-appearance-of-silver-nitrate-on-ct-imaging-in-patients-with-severe-otitisexterna-a-case-series 